\title{
Le magasin « Vuka Africa »
}

\section{Commerçants africains et pratiques culturelles en Afrique du Sud au temps de la ségrégation et de l'apartheid (années 1880 - 1960)}

\author{
Alan Cobley \\ Traduit de I'anglais par Laurent Chauvet et Sophie Dulucq
}

Citer cet article : Cobley Alan (2021), « Le magasin "Vuka Africa" Commerçants africains et pratiques culturelles en Afrique du Sud au temps de la ségrégation et de l'apartheid (années 1880 - 1960) 》, Revue $d^{\prime}$ Histoire Contemporaine de l'Afrique, $\mathrm{n}^{\circ} 2,66-88$, en ligne. URL :

https://oap.unige.ch/journals/rhca/article/view/02cobley

Mise en ligne : $1^{\text {er }}$ octobre 2021.

DOI : https://doi.org/10.51185/journals/rhca.2021.e553

\section{Résumé}

À partir de la fin du XIX siècle, la croissance urbaine rapide dans la région du Witwatersrand (Afrique du Sud) offre des perspectives d'avenir à une classe émergente de colporteurs et de camelots africains, ainsi qu'à des commerçants « indigènes » qui cherchent à s'établir de façon sédentaire dans les quartiers qui leurs sont réservés. Le présent article étudie comment cette catégorie sociale naissante a grandement contribué à l'émergence d'une culture noire urbaine dynamique, alors même que ces acteurs sociaux luttaient, au temps de la ségrégation et de l'apartheid, pour établir des commerces viables et pour combattre les mesures racistes visant à exclure les Noirs des villes, ou à les marginaliser. Ce faisant, leur participation a été importante dans le développement de nouvelles pratiques culturelles noires dans les villes, surtout sur le plan des modes de consommation.

Mots-clés : culture noire urbaine; alimentation; commerce ; commerçants ; ségrégation ; apartheid ; consommation 
À partir de 1886, dans la région du Witwatersrand, Johannesburg et d'autres centres urbains connaissent une croissance rapide sous l'effet du développement des activités d'extraction aurifère et des industries qui leur sont liées. Cette urbanisation donne naissance à une population citadine multiraciale, partagée entre un groupe en pleine croissance de résidents permanents et plusieurs dizaines de milliers de travailleurs immigrés de passage. Parmi ceux qui saisissent les opportunités nouvelles ainsi créées dans la première moitié du XXe siècle, émerge un ensemble éclectique constitué de colporteurs et de camelots africains, ainsi que de commerçants « indigènes » qui s'établissent comme « commerçants sédentaires » dans des quartiers séparés, d'abord appelés officiellement Native Locations, puis Black townships à partir des années 1950'.

À cette période, la principale loi ségrégationniste relative à la gestion des Africains dans les villes d'Afrique du Sud est le Native (Urban Areas) Act de 1923, qui connaîtra de nombreuses modifications ultérieures ${ }^{2}$. Cette loi donne aux autorités municipales le pouvoir de faire évacuer les Africains des zones résidentielles désignées comme blanches en procédant au " déguerpissement » des taudis et d'établir des «townships indigènes » pour héberger la population africaine permanente ainsi déplacée. Elle fournit par ailleurs un cadre juridique pour les administrer. Les conditions dans lesquelles les Africains peuvent devenir propriétaires de boutiques et entretenir des relations commerciales dans ces townships sont exposées à la section 22 de la loi de 1923.

En luttant pour établir en ville des commerces viables possédés par des Noirs, les commerçants africains ont contribué à ce qu'une nouvelle culture noire se développe, malgré les embûches, dans l'Afrique du Sud de la première moitié $d u X X^{e}$ siècle. Leurs magasins constituent en premier lieu des repères géographiques pour les communautés urbaines qu'ils desservent, mais ils deviennent aussi des lieux de rencontre. Ils servent également d'exemples et de modèles de modernité urbaine réussie. Enfin, leur succès économique dépendant d'une étroite symbiose avec les communautés environnantes, leur fonctionnement et les marchandises qu'ils vendent reflètent - tout en contribuant à les transformer - des modes de consommation associés à la vie en ville.

Ces commerçants ont suscité relativement peu d'attention dans I'historiographie des villes sud-africaines, la grande partie de cette littérature portant plutôt sur les luttes de la classe ouvrière noire urbaine et sur les difficultés matérielles qui lui sont associées (logement, transports, approvisionnement, assainissement, hygiène et loisirs). Lorsque les commerçants africains apparaissent dans les premières études sur les communautés urbaines - telles que celles dirigées par I'Institut sud-africain des relations raciales (SAIRR) -, ils focalisent rarement I'attention ${ }^{3}$. An African Bourgeoisie, ouvrage sociologique majeur de Leo Kuper publié en 1965,

\footnotetext{
${ }^{1}$ Cet article porte sur la portion proprement africaine des populations urbaines considérées comme noires en Afrique du Sud (Africains, Indiens, Métis), cette catégorie ayant par ailleurs une histoire distincte dans les zones urbaines au temps de la ségrégation et de l'apartheid. Les " commerçants sédentaires » sont des commerçants qui vendent dans des locaux loués, fournis à cette fin par les autorités municipales dans les zones urbaines réservées aux "indigènes » (Urban Native Locations). Ces zones ont, durant le régime d'apartheid, été ensuite désignées sous le terme générique de «black townships».

2 Cette loi a été modifiée en 1930, 1937, 1944, consolidée en 1945, puis modifiée à nouveau en 1945, 1946, 1947, 1952, 1955, 1956, 1957, 1963 et 1964. Voir : Davenport Rodney (1969), "African Townsmen? South African Natives (Urban Areas) Legislation through the Years ", African Affairs, 68(271), pp. 95-109.

${ }^{3}$ Le volume, par ailleurs détaillé, de Hellmann Ellen (dir.) (1949), Handbook on Race Relations in South Africa, Johannesburg, SAllR ne contient pratiquement aucune référence aux commerçants africains dans les zones urbaines.
} 
a été l'un des premiers travaux à offrir une analyse approfondie sur ces commerçants africains. Mais ils y sont considérés davantage du point de vue de leur classe sociale qu'à travers leur rôle dans les productions culturelles ${ }^{4}$. De même, comme l'indique Deborah Posel, "I'histoire de la consommation en Afrique du Sud reste à écrire dans son ensemble [...] ce qui laisse des vides significatifs dans l'histoire des groupes raciaux, de la règlementation raciale et de son articulation avec la "capacité à désirer" $"$ ». Comme l'explique Timothy Burke, cela est dû en partie au fait que « la production et la reproduction de la culture marchande [est] un processus qui brouille ses propres pistes » : «II est difficile d'enquêter pour savoir comment et quand un produit devient "nécessaire", alors même que tous, au sein d'une société donnée, semblent s'accorder sur l'idée que cette nécessité a toujours existé »6.

Cet article cherche donc à combler ces deux lacunes considérables dans l'historiographie de la culture noire urbaine en Afrique du Sud. Après avoir étudié le parcours à succès d'un commerçant, la présente contribution analysera l'implantation des commerces de détail africains à Johannesburg et, ce faisant, les transformations des habitudes de consommation en ville.

\section{Le magasin « Vuka Africa »}

Dans la soirée du 9 juin 1955, John Carlie Phillip Mavimbela, propriétaire du magasin «Vuka Africa » ("Debout l'Afrique ») dans l'Eastern Native Township", meurt au volant de sa Chevrolet dans un accident de voiture, sur la route qui relie Johannesburg à Vanderbijlpark ${ }^{8}$. II rentrait chez lui, dans le township africain d'Evaton, après avoir assuré la fermeture de sa boutique en début de soirée. Né à Johannesburg le $1^{\mathrm{er}}$ mai 1905, fils d'une lavandière et d'un ouvrier, Mavimbela appartient à l'une des premières générations d'Africains nés et élevés en ville. Les détails sur son enfance sont lacunaires, mais on sait que, de la fin des années 1920 à 1940, il a travaillé comme enseignant dans l'Eastern Native Township - années durant lesquelles il étudie par correspondance et obtient un diplôme universitaire (bachelor's degree). II arrondit alors ses fins de mois en jouant du saxophone alto le soir avec les Merry Blackbirds, premier orchestre noir de danse de Johannesburg. Il a voyagé au Royaume-Uni avec l'orchestre aux alentours de 1930 pour y réaliser des enregistrements pour le label Singer ${ }^{9}$. Parmi leurs nombreuses prestations mémorables, les Merry Blackbirds ont joué lors de la réception de

\footnotetext{
${ }^{4}$ Kuper Leo (1965), An African Bourgeoisie. Race, Class and Politics in South Africa, New Haven and London, Yale University Press.

5 Posel Deborah (2010), « Races to Consume: Revisiting South Africa's History of Race, Consumption and the Struggle for Freedom », Ethnic and Racial Studies, 33(2), pp. 157-175.

${ }^{6}$ Burke Timothy (1996), Lifebuoy Mean, Lux Women. Commodification, Consumption and Cleanliness in Modern Zimbabwe, Durham and London, Duke University Press, pp. 8-9.

7 Township établi dans une banlieue industrielle à l'est de Johannesburg.

${ }^{8}$ Mavimbela est mort d'une fracture du crâne. On raconte que l'autre conducteur, Leon Booysens, conduisait à grande vitesse du mauvais côté de la route lorsque les voitures sont entrées en collision. Booysens nie avoir causé l'accident, mais sa compagnie d'assurance paye $600 £$ à la veuve de Mavimbela, Julia, en règlement amiable, plutôt que de porter l'affaire devant les tribunaux : Archives nationales d'Afrique du Sud (NASA), TAB WLD Illiquid 1957 2124/57, « Julia Tompi Mavimbela vs Aetna Insurance Company Limited ». Le fils aîné de Mavimbela est mort d'un accident sur la même route en 1964.

9 Université du Witwatersrand, Department of Historical Papers (UW), « [Biography of] J. C. P. Mavimbela » de T.D. Mweli Skota, A1618, T. D. M. Skota Papers, File 12, « Notes on conversation between Mr Wilson Silgee and Alan Cobley, Soweto, $1^{\text {st }}$ November 1983 ». Les enregistrements des Merry Blackbirds sont liés à Ballantine Christopher (1993), Marabi Nights. Early South African Jazz and Vaudeville, Johannesburg, Ravan Press.
} 
mariage de Walter et Albertina Sisulu au Bantu Men's Social Centre (BMSC) en 1944 ; Nelson Mandela y était garçon d'honneur ${ }^{10}$.

Durant la Deuxième Guerre mondiale, Mavimbela quitte l'enseignement pour travailler pour le compte de la Young Men's Christian Association (YMCA) et du mouvement international chrétien Toc- $\mathrm{H}$, exerçant comme organisateur auprès des recrutés noirs des camps militaires du Witwatersrand. On lui délivre un certificat le dispensant des restrictions liées au couvre-feu imposé aux Noirs afin de faciliter son travail11. Après la guerre, Mavimbela acquiert une licence commerciale pour un "magasin général ${ }^{12}$ » et devient, pour un loyer mensuel de 9 livres et 10 shillings, locataire du magasin situé au $n^{\circ} 5$ de James Street, dans I'Eastern Native Township. Son affaire est vite florissante et, en 1947, il obtient également un permis pour ouvrir un restaurant; il dépense alors 8 livres pour convertir une partie de son local à cet usage ${ }^{13}$. Il épouse une jeune enseignante du nom de Julia Tompi Ngubeni en 1946 et le couple a très vite des enfants. Vers 1950, ils déménagent de l'Eastern Native Township - quartier pauvre où ils logeaient à l'étroit - dans une maison construite sur un terrain dont il est propriétaire, au 1381, Mikado Road ${ }^{14}$, dans le township d'Evaton - où les Noirs pouvaient disposer de propriétés foncières. La maison de briques est spacieuse et de qualité. Elle a une surface au sol de 1485 pieds carrés (environ $138 \mathrm{~m}^{2}$ ), sans compter la véranda. Elle dispose de six pièces, d'une cuisine, d'une salle de bain et d'un garde-manger, et elle est entièrement raccordée au réseau électrique. Elle a également un garage et une réserve. À la mort de Mavimbela, elle est estimée à 2425 livres.

Mavimbela prend une part active à la vie politique locale. À partir de 1935, il est à plusieurs reprises membre du comité consultatif de l'Eastern Native Township ${ }^{15}$. De 1935 à 1937, il représente également l'Eastern Native Township lors du Congrès des comités consultatifs des townships établi par R. H. Godlo, un commerçant d'East London. II préside le comité d'organisation du pique-nique annuel des enfants de l'Eastern Native Township et héberge cet événement annuel de 1940 jusqu'à sa mort en $1955^{16}$. À son décès, il est en train d'œuvrer avec deux autres hommes d'affaires importants, Paul Mosaka et S. J. J. Lesolang, à la création d'une organisation visant à réunir les entrepreneurs africains de Johannesburg : la Chambre de commerce africaine ${ }^{17}$. Cette structure sera l'embryon de la première grande organisation nationale regroupant les hommes d'affaires africains en Afrique du Sud, fondée

\footnotetext{
10 Odendaal Andre (2003), The Story of an African Game. Black Cricketers and the Unmasking of One of South Africa's Greatest Myths, 1850-2003, Cape Town, David Philip, p. 94. D'autres dirigeants africains éminents étaient présents au mariage, y compris le président de I'ANC, le Dr A. B. Xuma, ainsi qu'Oliver Tambo et Anton Lembede.

11 UW, «[Biography of] J. C. P. Mavimbela ». L'organisateur principal du YMCA «non européen » durant cette période était J. R. Rathebe, qui avait été détaché de son poste de secrétaire du BMSC durant la guerre : Cobley Alan (1997), The Rules of the Game. Struggle in Black Recreation and Social Welfare Policy in South Africa, Westport, Greenwood, pp. 147 et 160, note 86 ; NASA, SAB URU 189032208 Dossier 2167/ 2243, « Letters of Exemption » for JCP Mavimbela issued 26, 1. 40.

${ }^{12}$ Commerce offrant une large variété de produits, de l'alimentaire aux produits manufacturés de consommation courante.

13 Report of the Native Affairs Committee 25 mars 1947 in « Minutes of the Meetings of the Johannesburg City Council, JanuaryJune $1947 »$.

${ }^{14}$ Dans la même rue que l'école de formation de l'Église épiscopale méthodiste africaine, l'Institut Wilberforce.

15 Des comités consultatifs avaient été établis sous la Loi de 1923 sur les Zones urbaines indigènes, dans le cadre d'un mécanisme consultatif entre les résidents des townships et les autorités municipales.

${ }^{16}$ UW, AD843/R, J. D. Rheinallt Jones, South African Institute of Race Relations Papers, dossier «Organisations », J. C. P. Mavimbela to Secretary, SAIRR, 11 novembre 1940.

17 Mavimbela était l'un des responsables à avoir convoqué la réunion initiale, tenue dans la salle des fêtes de l'Eastern Native Township, le 7 mai 1955 : "African Traders Meeting at E.N.T », Bantu World, 7 mai 1955. L'association a été officiellement créée peu de temps après sa mort.
} 
en 1964 : la Fédération nationale des chambres de commerce africaines (NAFCOC, National African Federated Chambers of Commerce) ${ }^{18}$.

Les finances du magasin «Vuka Africa » sont en bonne santé au moment de la disparition de Mavimbela. Les livres de comptes détaillés, tenus par sa femme Julia, indiquent que, durant les quatre années précédant son décès, ses affaires ont généré un bénéfice brut d'environ 2000 livres par an, avec des bénéfices nets annuels oscillant entre 500 et 600 livres. En 1952, les coûts se décomposent ainsi : loyer (£138), salaires (£1140), transport (£180), frais divers $(£ 24)$.

L'inventaire après décès offre un instantané du stock que possède, dans les années 1950, un commerçant prospère établi dans un township:

Tableau $n^{\circ} 1$ : inventaire des stocks du magasin « Vuka Africa »19

\begin{tabular}{|c|c|c|}
\hline Quantité & Article & Valeur \\
\hline 2 boîtes & Savon Sunlight & $£ 3.1 .10$ \\
\hline 6 douzaines & Paillettes Lux ${ }^{20}$ & $£ 3.2 .1$ \\
\hline 3 douzaines & $\operatorname{Vim}^{21}$ & $£ 1.11 .0$ \\
\hline 1 douzaine & Milo 22 & $£ 1.7 .6$ \\
\hline 4 douzaines & «Corned-beef » & $£ 3.15 .0$ \\
\hline $41 / 2$ douzaines & Haricots sauce tomate & $£ 1.13 .9$ \\
\hline 21 & Bougies & $£ 1.2 .9$ \\
\hline 44 & Tasses et soucoupes & $£ 3.13 .4$ \\
\hline 10 douzaines & Cire à parquets & $£ 10.8 .4$ \\
\hline 4 sacs & Farine de maïs & $£ 1.14 .0$ \\
\hline 17 paquets & Players $^{23}$ & $£ 0.19 .8$ \\
\hline \multirow[t]{2}{*}{10 paquets } & Filter Tip Commando ${ }^{24}$ & $£ 2.2 .8$ \\
\hline & Total du stock évalué à & $£ 245.16 .2^{25}$ \\
\hline
\end{tabular}

\footnotetext{
18 Lesolang est un ancien enseignant. II gère la plus grande concession d'Orlando. Mosaka est titulaire d'un diplôme universitaire et, parmi d'autres activités commerciales, a créé le premier funérarium dans les townships de Pimville.

19 NASA TAB MHG 4256/55. "Estate of the late John Carlie Phillip Mavimbela. Surviving Spouse Julia Nompi".

20 Marque de lessive.

21 Marque de détergent.

22 Marque de boisson cacaotée en poudre.

23 Marque de cigarettes.

24 Filtres à cigarettes.

25 «245.16.2 » se lit : 245 livres, 16 shillings et 2 pence. Jusqu'au début des années 1960, on utilise en Afrique du Sud le système britannique de comptabilité qui décompose les sommes d'argent en livres, shillings et pence. II y a 20 shillings dans 1 livre et 12 pence dans 1 shilling.
} 
Les conserves et les produits de nettoyage inclus dans l'inventaire témoignent des changements survenus dans les habitudes alimentaires et les modes de consommation des Africains en ville. Quant aux cigarettes, elles renvoient à l'importante consommation de tabac, quasi omniprésente à l'époque chez les hommes noirs des villes ${ }^{26}$. L'inventaire détaille également les équipements du magasin - une scie de boucher, des vitrines d'exposition, trois tables, quinze chaises et un réfrigérateur - évalués à 240 livres et 15 shillings. II ne fait aucun doute que le mobilier servait dans le restaurant situé à côté du magasin ; la scie de boucher et le réfrigérateur permettaient de fournir de la viande fraîche à la clientèle. Comme l'explique Ellen Hellmann dans un article consacré à « l'alimentation indigène urbaine » en 1936, la viande constitue alors un élément beaucoup plus présent dans l'alimentation des urbains que dans celle des ruraux :

L'Indigène a toujours montré une préférence marquée pour la viande, et de nombreuses femmes m'ont bien expliqué que l'un des avantages de la vie en ville est la possibilité de pouvoir acheter des petites quantités de viande quotidiennement. ${ }^{27}$

Dans son testament, Mavimbela laisse à son épouse Julia le magasin et le gros de la succession, pour un ensemble évalué à 4700 livres, 8 shillings et 2 pence. La succession inclut deux propriétés à Evaton, une à Claremont (Durban) et une somme de $600 £$ déposée à la banque Barclays de Jeppestown. Dans le contexte de pauvreté persistante dans les townships noirs, tout cela suggère une accumulation de richesses remarquable. Toutefois, la gestion difficile d'un commerce de township apparaît au détour du commentaire que fait la comptable qui évalue la succession :

[À] mon avis, la valeur du fonds de commerce de feu J.C.P. Mavimbela, exploité sous le nom de Vuka Africa au 5 James Avenue, Eastern Native Township, est nulle. Je suis de cet avis car je constate qu'il n'y a aucune garantie d'occupation, les locaux étant situés dans l'Eastern Native Township et faisant l'objet d'une location mensuelle... [...] En outre, en cas de vente de l'entreprise, le locataire n'est pas en mesure de transmettre les droits de location à l'acheteur, qui devra demander au conseil municipal de Johannesburg, en concurrence avec d'autres candidats éventuels, l'autorisation d'exercer l'activité et de louer le local commercial au mois. ${ }^{28}$

Cette évaluation pessimiste ne prend pas en compte la détermination et l'ingéniosité de la veuve de Mavimbela, Julia, ni la loyauté de la clientèle du magasin. Julia persuade les autorités municipales de l'autoriser à reprendre la boutique et la licence commerciale de son mari ; elle s'impose rapidement comme une femme d'affaires accomplie, alors même qu'elle doit s'occuper de ses cinq enfants en bas âge. En janvier 1956, le journal The World rapporte qu'elle a transformé le pique-nique annuel organisé par son mari en une fête du Jour de l'An,

\footnotetext{
26 Pour une discussion sur l'établissement et l'adoption généralisée des savons, tels que Sunlight et Lux, comme produits de base chez les Africains en Afrique australe, voir Burke T., Lifebuoy Men, Lux Women..., op. cit., pp. 92-99 et 153-155. Bien que les données historiques sur le tabagisme soient limitées, une enquête a permis d'établir que, jusqu'en 1975-1976, 70\% des hommes africains fumaient des cigarettes en Afrique du Sud.

27 Hellmann Ellen (1936), « Urban Native Food in Johannesburg », Africa, 9(2), pp. 277-290.

28 Certificat signé par Krishna Govinda Samy Moodley (comptable), daté du 2 juillet 1956, joint à la « Succession du défunt John Carlie Phillip Mavimbela. Épouse survivante Julia Nompi ».
} 
tenue au magasin pour les "gamins du township », avec cadeaux sous l'arbre de Noël et des rafraîchissements servis à la boucherie, dans un local voisin. L'article commente ainsi :

Madame Mavimbela gère les trois commerces que lui a laissés son mari : un restaurant, une épicerie et une boucherie. Elle s'occupe de la comptabilité, passe les commandes et supervise les ouvriers [...] Peu d'entre nous seraient capables d'assumer de telles responsabilités comme le fait Mme Mavimbela, qui constitue un exemple pour nous tous. ${ }^{29}$

Dans un portait de Madame Mavimbela publié six mois plus tard, il est indiqué que sa philosophie est de « prendre les choses comme elles viennent et de s'en accommoder du mieux que l'on peut ${ }^{30} »$. Après un début de carrière d'enseignante dans les écoles rurales, elle est sortie diplômée de l'Institution Kilnerton en 1937 et a enseigné à l'École méthodiste de Springs, avant de recevoir une bourse pour passer un certificat d'enseignement de maternelle et de primaire supérieur. Elle a commencé par enseigner dans une classe de maternelle à l'école méthodiste de la rue Albert, à Johannesburg. Elle est ensuite nommée au poste de directrice de l'école primaire de Boksburg en 1946, I'année de son mariage avec John Mavimbela. Toutefois, "[l]orsqu'elle voit son mari prêt à capituler devant les soucis liés à son commerce, elle démissionne de son poste pour lui apporter son soutien moral ». Elle explique au journal : « Je suis extrêmement heureuse de cette décision, car cela m'a permis me familiariser avec les affaires commerciales $»^{31}$.

Les commerçants africains de l'époque vivent dans la peur constante du chapardage commis par leurs employés sous-payés, préférant souvent fermer le magasin lorsqu'ils doivent quitter les locaux, de sorte que les heures d'ouverture des magasins peuvent être irrégulières et imprévisibles. B. J. Mabuza explique ainsi au journal The World qu'« un homme d'affaires ne peut faire confiance à personne »:

Une pièce de trois pence pour moi, une pièce de six pence pour toi. Une pièce de six pence pour moi, une pièce de douze pence pour toi. Telle est la politique de la plupart des employés de commerce africains. Et il semble qu'il n'y ait aucune manière de surveiller cela. Un homme d'affaires ne peut même pas faire confiance à sa femme à cet égard. ${ }^{32}$

De toute évidence, Mabuza n'a pas été aussi chanceux dans le choix de son épouse que ne l'a été John Mavimbela : il est clair que la décision de Julia de travailler à plein temps au magasin a contribué à sa réussite.

Leur commerce étant florissant, les Mavimbela sont capables de générer des profits suffisants pour faire des dons au sein du township, notamment aux organisations religieuses et aux clubs d'enfants. Ils sont également parrains du club de football « George Goch Eastern

\footnotetext{
29 «Women's World: Brave Wife Carries on Husband's Good Work », The World, 11 janvier 1956.

30 «Africans Can Do It. Mrs Mavimbela Continues the Work of Her Husband », The World, 6 juin 1956.

31 Ibid.

32 «Never, Never Trust a Soul! - Businessman», The World, 2 mai 1956. B. J. Mabuza a commencé dans le commerce comme «épicier et fruitier » dans Market Street, à Johannesburg, en 1924. En 1927, il est le premier Africain à obtenir un permis de restauration à Sophiatown, mais il vend son affaire à un Blanc en 1951, «à un moment où une rumeur insistante parle d'envoyer les hommes d'affaires africains dans les townships ». Ibid.
} 
Brothers » et du groupe musical de Ramokgopha, les Lo-Six ${ }^{33}$. Ces investissements dans la vie sociale et culturelle du township ne constituent pas de simples actes philanthropiques: ils contribuent à forger un esprit de communauté et à fidéliser la clientèle du magasin.

Le cas spécifique du magasin «Vuka Africa » illustre bien les efforts des commerçants africains qui, dans la première moitié $d u X x^{\mathrm{e}}$ siècle, créent et font vivre des commerces prospères et bien intégrés dans les communautés noires urbaines. Il laisse également entrevoir le rôle que ces commerces ont joué dans la formation d'une vie culturelle noire, partie intégrante des productions culturelles qui émergent dans les villes au temps de la ségrégation et de l'apartheid. Nous allons maintenant étudier, dans un contexte historique plus large, leur rôle dans ces productions culturelles.

\section{Les origines des commerces africains dans les zones urbaines}

En l'espace d'à peine quarante ans, la population urbaine africaine quadruple en Afrique du Sud, passant d'un peu plus d'un demi-million en 1911 à 2240000 en 1951. C'est en 1946 que le nombre des Africains dans les zones urbaines dépasse pour la première fois celui des Blancs $^{34}$. Au fur et à mesure que cette population augmente, les commerces noirs se multiplient pour pourvoir à la consommation des Africains travaillant en ville ainsi qu'au flux constant de travailleurs immigrés. Nombre de ces activités commerciales sont assurées par des colporteurs et des camelots, qui mènent leurs opérations à très petite échelle, vendant des produits frais, des bibelots, des allumettes, du tabac et divers articles dans leurs charrettes à bras, ou qui se sont établis comme « artisans indigènes » à leur compte, offrant au coin des rues des services tels que la coiffure pour hommes ou la réparation de chaussures. Jusqu'aux années 1930, certains artisans - par exemple des charpentiers, des couturiers et des cordonniers - vivent et travaillent également dans des pièces qu'ils louent dans les quartiers très défavorisés. D'après Rogerson, les "café-de-move-ons ${ }^{35}$ " ou "charrettes à café » ont commencé à faire leur apparition dans les rues de Johannesburg à la fin des années 1920; on y vend au départ du thé, du café et des petits gâteaux à un penny pièce. En 1933, on comptabilise 155 camelots vendant de la nourriture dans les rues : ce chiffre atteint son plus haut niveau en 1955, avec environ 1000 commerçants de ce type. En 1964, le Rand Daily Mail évoque les «milliers d'Africains déjeunant chaque jour sur les trottoirs et dans les caniveaux » de la ville ${ }^{36}$. Jusqu'au milieu des années 1950, le menu d'une « charrette à café » typique inclut les denrées suivantes:

\footnotetext{
33 Les résidents connaissent également l'Eastern Native Township sous le nom de "George Goch », du nom d'un ancien maire de Johannesburg et de la gare ferroviaire voisine.

34 South Africa, Bureau of Census and Statistics (1960), Union Statistics for Fifty Years, Pretoria, Bureau of Census and Statistics.

${ }^{35}$ Ce terme un peu énigmatique - mélange de français et d'anglais - désignait localement de petits stands à café sur roues.

${ }^{36}$ Cité dans Rogerson C. M. (1986), "Feeding the Common People of Johannesburg, 1930-1962 », Journal of Historical Geography, 12(1), pp. 56-73.
} 
Tableau $n^{\circ} 2$ : Denrées d'une « charrette à café » $^{37}$

\begin{tabular}{|l|l|}
\hline Thé ou café & 3 pence la grande tasse \\
\hline Pain, sec & 1 penny la tranche \\
\hline Pain, beurré & 3 pence la tranche \\
\hline Vetkoek $^{38}$ & 1 penny chacun \\
\hline Boisson de farine de maïs fermentée & 3 pence la conserve d'une livre \\
\hline Lait fermenté & 1 shilling et 9 pence le bol \\
\hline Ragoût de viande & 1 shilling l'assiette \\
\hline Bouillie de farine de maïs & 1 shilling l'assiette \\
\hline
\end{tabular}

D'autres commerces importants dont les propriétaires sont Africains ont également commencé à faire leur apparition en ville. On y trouve notamment des restaurants " cafres » ou « indigènes », datant des premières prospections minières dans la région durant les années 1880, et qui sont rapidement devenus, pour les Africains, emblématiques de la vie urbaine. En 1904, on comptabilise 52 « restaurants indigènes » à Johannesburg. Ce chiffre monte à 174 en 1936, dont 144 appartenant à des Blancs, 17 à des « Asiatiques » (principalement Chinois) et 17 à des Africains ${ }^{39}$. Peter Abrahams se souvient avoir mangé dans les années 1930 dans un « restaurant indigène », The Burning Meat, à Vrededorp :

Les gars du marché allaient là-bas chaque fois qu'ils pouvaient se payer le prix d'un repas. Pour trois pence, on pouvait obtenir un morceau de viande nageant dans un bouillon clair et huileux, avec un gros morceau de pain. L'endroit lui-même appartenait aux mouches. Nous, les humains, nous nous y trouvions par tolérance. Les mouches marchaient sur le sol couvert de résine, copulaient sur les longs bancs et les longues tables où s'asseyaient les humains, noircissaient le plafond et opacifiaient la fenêtre. ${ }^{40}$

Selon Ellen Hellmann, trois types de « restaurants indigènes » existaient à Johannesburg dans les années 1930. Les plus simples étaient ceux que l'on pouvait trouver dans les locaux commerciaux des concessions minières, ouverts à une clientèle $d^{\prime}$ ' indigènes employés dans les mines ». En ville, deux types de restaurants étaient fréquentés par les Africains travaillant hors secteur minier: «un type grossier et rudimentaire ${ }^{41}$ » et « un type plus raffiné où la vaisselle [était] de meilleure qualité et les prix plus élevés ${ }^{42}$ ». Parmi ces restaurants plus raffinés, on peut mentionner celui que gère E. P. Moretsele au Market Square, des années 1920 aux

\footnotetext{
37 Information compilée par le Service de Santé publique de Johannesburg, cité par Rogerson C. M., « Feeding the Common People... », art.cité, p. 67.

38 Pain frit garni.

${ }^{39}$ Rogerson C. M.., p. 57, cite le Journal officie/ du 4 mars 1904 : « L'expression "restaurant cafre" désigne les lieux et locaux où des articles de nourriture ou de boisson sont vendus ou offerts à la vente aux indigènes, et où un espace est mis à disposition pour la consommation de ladite nourriture ou boisson »; Hellmann E., « Urban Native Food in Johannesburg... », art. cité, p. 24.

40 Abrahams Peter (1954), Tell Freedom, Londres, Faber \& Faber, pp. 185-186.

41 Ibid.

42 Hellmann E., « Urban Native Food in Johannesburg... », art. cité, p. 286.
} 
années 1950. L'établissement offre ce que l'on pourrait qualifier de consommation ostentatoire, destinée à une clientèle noire appartenant à la « classe moyenne »; il devient un lieu de rencontre prisé des membres du Congrès National Africain (ANC) ${ }^{43}$. Sur la question de la «consommation ostentatoire", Deborah Posel note que "les modes de consommation individuels font partie intégrante du sens de soi et de la sociabilité - et constituent donc un espace important d'exercice du pouvoir ${ }^{44} »$.

\section{Commerçants blancs, indiens, chinois et commerce « indigène »}

Au début du $x^{e}$ siècle, dans les villes telles que Johannesburg la plupart des magasins pourvoyant aux besoins des « indigènes » appartiennent à des hommes d'affaires juifs, indiens ou libanais ${ }^{45}$. Avant la Première Guerre mondiale, ces groupes d'immigrants se sont établis dans le Witwatersrand en marge de l'Afrique du Sud «blanche » et de la majorité de la population africaine. N'étant ni complètement acceptés, ni intégrés dans l'une ou l'autre communauté, ils se sont naturellement tournés vers l'espace socio-économique intermédiaire qu'offre le " commerce indigène ». Ils vendent une grande gamme d'articles: des couvertures, des vêtements, des chaussures, des batteries de cuisine, ainsi que des produits frais, de la farine de maïs ou des conserves. Leurs magasins se regroupent près de l'entrée des complexes miniers, aux portes des premiers « townships indigènes » urbains ou le long des routes reliant le centreville aux quartiers pauvres, où vivent des populations diverses sur le plan racial.

Un article publié dans la première édition de Ilanga lase Natal( The Sun of Natah), en avril 1903, présente ainsi ces « magasins cafres » situés dans les concessions minières Johannesburg et de sa périphérie ${ }^{46}$ :

Ce commerce se développe désormais rapidement car, pour la première fois, le Cafre touche un bon salaire et le dépense. Les marchandises vendues dans ces magasins sont principalement de marques américaines et allemandes. On y trouve des tissus de coton bleu imprimé - les imprimés allemands étant les plus appréciés, car leurs motifs sont imprimés des deux côtés ou le dos du matériau est foncé. Les malles et les boîtes sont désormais fabriquées en Amérique et privilégiées, car les serrures en sont beaucoup plus robustes et que, sur chaque boîte, on en trouve généralement deux au lieu d'une. On trouve aussi des châles imprimés: les Allemands en fabriquent de plus doux que les Anglais ; les châles allemands sont plus chers, mais pas au point que le Cafre ne puisse payer la différence. Il y a aussi des parfums, des tabatières et des articles sophistiqués venus principalement d'Allemagne - aujourd'hui, le Cafre a de l'argent à dépenser

\footnotetext{
43 Elias Moretsele était originaire du Transvaal du Nord-Est. Jeune homme, il établit son commerce après avoir déménagé à Johannesburg. II a rejoint le Congrès en 1917, sert de trésorier provincial dans les années 1940, et succède à Nelson Mandela comme président de I'ANC du Transvaal dans les années 1950. Karis Thomas et Carter Gwendolyn (1977), From Protest to Challenge. $A$ Documentary History of African Politics in South Africa 1882-1964, 4, Stanford, Hoover Institution Press, p. 97.

44 Posel D., « Races to Consume... », art. cité, p. 162.

45 Les juifs ashkénazes ont massivement émigré de l'Europe de l'Est vers l'Afrique du Sud des années 1880 à 1914, et à nouveau entre 1920 et 1930. Durant cette période, "peu de localités du Cap, de l'État Libre d'Orange, et plus tard du Transvaal, n'ont pas leurs camelots ou leurs commerçants juifs, généralement assez vite rejoints par leurs familles et des parents restés au pays. En ligne, URL : https://www.jewishvirtuallibrary.org/south-africa (Consulté le 30 octobre 2020). Les immigrants syro-libanais arrivent à la même époque : «À leur arrivée, la plupart ont d'abord travaillé dans les mines, puis se sont reconvertis dans le commerce de produits frais, ou bien sont devenus colporteurs de marchandises ». Hourani Guita (2015), «The Struggle of the Christian Lebanese for Land Ownership in South Africa ». En ligne, URL : http://maroniteinstitute.org/MARI/JMS/july00/The_Struggle.htm (consulté le 30 octobre 2020).

${ }^{46}$ L'expression « cafre » (kafir ou kaffir) était largement utilisée par les Blancs pour parler des Noirs en Afrique du Sud jusqu'au début du $x x^{e}$ siècle, mais elle a graduellement été remplacée par le terme « indigène » considéré comme moins offensant.
} 
pour des bagatelles - et des costumes pour Cafres en épais tissu à carreaux - ces costumes font l'objet d'un commerce énorme, et tout vient d'Amérique... des couvertures en coton ; le Cafre les aime bien épaisses, presque comme du feutre ; actuellement, elles viennent d'Allemagne. ${ }^{47}$

Cette description offre un précieux aperçu de la gamme de marchandises que les Africains achètent alors, et du discernement dont ils font preuve en les sélectionnant. Un auteur africain, qui n'est pas nommé dans /langa (il s'agit très certainement de John L. Dube, rédacteur du journal et futur président fondateur de I'ANC), commente ainsi :

Nous les indigènes, nous nous sommes souvent posés la question de savoir pourquoi les Européens ne se lancent pas davantage dans le commerce indigène. Les Arabes [Libanais] sont actifs dans ce commerce avec les indigènes, et font tout pour les persuader d'acheter. De façon générale, les indigènes craignent d'entrer dans le magasin d'un homme blanc, parce que les employés leur témoignent rarement de la bienveillance. Les femmes indigènes respectables se font généralement insulter, ce qu'elles ne supportent pas. Le commerçant les appelle "Mary », "Jane », ou tout autre nom les associant à des femmes de mauvaise réputation. ${ }^{48}$

II conseille vivement aux commerçants blancs de saisir l'occasion de mettre à profit la forte croissance de ce " commerce indigène », en plaçant des encarts publicitaires pour leurs magasins dans des journaux africains tels qu'llanga, et en "traitant les clients africains avec plus de respect ${ }^{49} »$.

Bien que la concurrence entre commerçants de toutes races soit féroce, la compétition entre non-Africains et Africains est exacerbée par les mesures discriminatoires imposées par les autorités. En 1931, dans la région du Witwatersrand, le seul township où I'on ne trouve pas de commerces pour Africains se trouve à Randfontein. D'après un membre du Comité consultatif local, «[c]ette situation est imputable aux commerçants juifs, qui influencent le Comité pour qu'il refuse de tels permis. Les indigènes se battent en vain pour ces droits depuis seize ou dix-sept ans déjà ${ }^{0}$ ». À la même époque, Cronje Bynight Mbolokwe, un boucher de Marabastad - un township de Pretoria - explique à la Commission économique indigène (NEC, Native Economic Commission) :

On interdit aux indigènes de bâtir comme ils le veulent, mais on donne le droit aux Indiens et aux Chinois de construire des grands bâtiments en brique. Les Indiens et les Chinois ne laissent aucune chance aux commerçants indigènes parce qu'ils sont bien organisés. Ils vendent à très bas prix afin de maintenir les indigènes dans un statut inférieur. ${ }^{51}$

\footnotetext{
47 "Trade with the Kafirs », article du Natal Mercury, repris dans Ilanga Lase Natal (The Sun of Natal), 1(1), 10 avril 1903.

48 Commentaire sur l'article « Trade with the Kafirs ».

49 lbid.

50 Témoignage de Meshach Nolisa Padi, Minutes of Oral Evidence Before the Native Economic Commission. Renumbered, vol. 11, «Johannesburg, Pretoria », 1931, p. 9220.

51 Témoignage de Cronje Bynight Mbolokwe, ibid, p. 8156. Mbolokwe est I'un des deux représentants de la section de I'ANC de Pretoria à comparaître devant la Commission. Il est accompagné de Simon Petros Matseke, un cordonnier qui a, par la suite, occupé le poste de président de l'ANC dans le Transvaal.
} 
Ezekiel Mphahlele se souvient des magasins chinois et indiens de Marabastad:

Les trois magasins chinois le long de Barber Street étaient des édifices en tôle mal construits, y compris ceux en face de chez nous qui appartenaient au riche Fung Prak. II s'agissait essentiellement d'épiceries et elles étaient en grand désordre. Les cinq magasins indiens consistaient en des bâtiments plus grands, en brique. Ils étaient bien rangés et chaque magasin avait un rayon pour l'épicerie, et un autre pour les tissus. Dans la véranda de tous ces magasins, le sol était paillé de coques de cacahuètes. Abdool possédait l'un de ces cinq magasins. Dans sa grande vitrine étaient exposés des bananes, des oranges, des fruits de la passion et des kujavasc'est ainsi que nous appelions les goyaves. Dans l'autre vitrine, on pouvait voir de la vaisselle pleine de poussière, des masques en papier et des petits jouets. ${ }^{52}$

La clientèle africaine qui se fournit chez les Indiens et les Chinois est souvent méfiante vis-à-vis d'eux et accepte mal un succès qui, de son point de vue, s'est fait au détriment de la communauté noire. Mphahlele se souvient d'un épisode où une dispute éclate entre sa tante Dora et le commerçant Indien Abdool, qui a refusé de tamponner son « livret de Noël » - une « carte de fidélité » rudimentaire qui, une fois entièrement remplie, donne droit à une tasse et une soucoupe ou à un autre petit cadeau - pour un achat totalisant les dix shillings: "Non, non, non, ah ah ah! » s'écrie Abdool, comme si l'idée lui était impensable. "C'est dix shillings de trop, de trop ! » [lui répond ma tante Dora] « Moi, je te dis de tamponner ce livret, coolie! Tu viens d'Inde pour te faire de l'argent sur notre dos, hein $! »^{53}$.

Le récit de Mphahlele à propos de la tension entre résidents des townships et commerçants indiens est raconté sur un ton humoristique, mais les tensions sont réelles et toujours prêtes à faire surface. À Durban, en janvier 1949, des émeutes sont déclenchées lorsqu'un jeune Africain est agressé par un commerçant indien et par son vendeur : elles font 142 morts et 1087 blessés ${ }^{54}$. La Commission d'enquête évoque l'« exploitation des indigènes par les commerçants indiens » et la «concurrence économique entre Indigènes et Indiens » parmi leurs causes premières ${ }^{55}$. C'est d'ailleurs à Durban que les tensions sont les plus fortes et qu'on trouve le plus fort taux de commerçants indiens par rapport au reste du pays. Le profond sentiment d'être leur victime est manifeste dans les remarques de Robert Sobukwe prononcées lors de la réunion inaugurale du Congrès Panafricain en avril 1959. Bien qu'il considère les SudAfricains indiens comme une minorité opprimée, il déclare que «la classe des marchands indiens [...] s'identifie en grande part avec l'oppresseur ${ }^{56} »$.

Une génération plus tard, durant les émeutes de Soweto, Mark Mathabane est témoin à Alexandra d'attaques contre des magasins indiens et chinois commises par de jeunes Noirs en colère :

Un matin, j'ai suivi une foule qui se déplaçait dans le ghetto, brûlant et pillant les magasins et les boucheries appartenant à des Indiens et des Chinois, dont tout le monde pensait qu'ils étaient devenus riches en dupant les paysans noirs et en les faisant payer trop cher. Une de ces familles

\footnotetext{
52 Mphahlele Ezekiel (1959), Down Second Avenue, Londres, Faber \& Faber, p. 53.

53 Ibid, pp. 108-109.

54 South Africa, Report of the Commission of Enquiry into Riots in Durban, Pretoria, Government Printer UG36-1949, p. 5: "Immediate

Cause of the Riots".

55 Ibid, pp. 15-16.

56 En ligne. URL : https://www.sahistory.org.za/archive/robert-sobukwe-inaugural-speech-april-1959 (consulé le 2 août 2021 ).
} 
chinoises - celle pour laquelle j'avais travaillé - était propriétaire de plusieurs magasins et boucheries dans la $12^{\mathrm{e}}$ Avenue. La famille s'était enfuie le premier jour des émeutes [...]. Un instant, j'ai pris conscience de l'absurdité de ce que nous faisions. Mais ces doutes ont laissé place à l'euphorie quand j'ai vu des paysans noirs repartir avec leur butin. Je les ai rejoints. ${ }^{57}$

\section{"Les ventes se comptent en pence »: Commerçants africains, pauvreté et modèles de consommation dans les communautés noires urbaines}

Le commerce sédentaire pour Africains a été autorisé à Johannesburg par plusieurs arrêtés municipaux, à la suite de l'établissement du premier township indigène urbain de la ville à Klipspruit en 1904. Par la suite, pour ce qui concerne le commerce, la règlementation municipale relative aux townships indigènes s'est modelée sur les dispositions de la loi de 1923 relative aux « Zones urbaines indigènes ${ }^{58} »$.

L'un des premiers commerçants à établir un magasin à Klipspruit est Thomas Ntlebi, originaire du Cap oriental. Après avoir validé un niveau scolaire de niveau III (ce qui équivaut à cinq années d'enseignement élémentaire) dans des écoles missionnaires, Ntlebi réussit l'examen d'élève-instituteur à l'Institut de formation indigène de Healdtown. En 1896, il travaille comme gérant dans un magasin de Fordsburg appartenant à un Blanc, commerce destiné à une clientèle principalement africaine. II crée ensuite sa propre boutique à Vrededorp puis, en 1905, ouvre un commerce à Klipspruit, qu'il dirige encore vingt-cing ans plus tard. Durant la période, il acquiert plusieurs propriétés à Kliptown et à Evaton, et devient également un membre éminent de l'« ordre anglican d'Éthiopie ». D'après Skota, c'est « l'un des pionniers africains du commerce dans le Transvaal 59 ». Le beau-père de Ntlebi est pasteur de l'Église méthodiste primitive wesleyenne, tandis que son beau-frère, J. Gqosho, travaille comme " receveur des postes » et gère une épicerie à Klipspruit ${ }^{60}$. Ces connexions familiales suggèrent que le commerce sédentaire est alors perçu par les Africains comme une occupation «prestigieuse » dans des townships tels que celui de Klipspruit.

Ce statut "d'élite » est également évident dans la famille de Phillip Merafe, un autre commerçant important de la génération suivante, établi à Pimville - nouveau nom de Klipspruit après 1934. Diplômé de Healdtown et de Lovedale, Merafe dirige un "magasin général » près de la gare ferroviaire. Il exerce pendant de nombreuses années la fonction de conseiller en chef du Comité consultatif du township. Lorsque sa fille, Ellen, déménage à Orlando pour y exercer comme enseignante en 1947, elle reçoit l'assistance du réseau de commerçant locaux pour s'établir. Elle raconte :

Grâce à ce réseau, j'ai su m'habiller d'une manière similaire à celle de mes collègues, ce qui m'a permis d'avoir davantage confiance en moi et d'avoir plus de dignité, et je suis devenue moimême une personne indépendante. ${ }^{61}$

\footnotetext{
57 Mathabane Mark (1986), Kaffir Boy. The True Story of a Black Youth's Coming of Age in Apartheid South Africa, New York, Free Press, pp. 264-265.

58 Copie déposée à la bibliothèque de l'université du Witwatersrand, Johannesburg, Native Location Regulations [Framed under section twenty-three (3) of the Natives (Urban Areas) Act of 1923], Johannesburg, Locations Department, 6 mars, 1925, pp. 8-9 et 13.

59 Skota Trevor Dan Mweli (dir.) (c. 1930), The African Yearly Register. Being an Illustrated National Biographical Dictionary (Who's Who) of Black Folks in Africa, Johannesburg, R. L. Esson \& $C^{\circ}$, p. 228.

60 Ibid, p. 153.

${ }^{61}$ Kuzwayo Ellen (1985), Call Me Woman, London, The Women's Press, p. 138.
} 
D'après des éléments recueillis par la Native Economic Commission (NEC), on trouve en 1932 dans les townships du Witwatersrand, quelque 500 « magasins indigènes », « les nombres les plus élevés étant atteints à Nancefield - quartier de Klipspruit - (40), Germiston (28) et Benoni (22). II y en a environ 45 dans le township d'Alexandra ${ }^{62}$. Toutefois, un administrateur local indique à la NEC que, même si la plupart des commerçants africains gagnent leur vie, « [il] n'en [connaît] aucun que l'on [puisse] considérer comme prospère ${ }^{63}$ ». Un des problèmes rencontrés est celui de la garantie des achats auprès des grossistes, comme l'explique Cronje Mbolokwe, établi à Pretoria, lors d'un échange avec les commissaires :

Les affaires des commerçants indigènes ne marchent pas bien. Ils ne sont pas protégés contre les achats excessifs. Aucun système n'est négocié entre grossistes et commerçants indigènes. On ne fait pas confiance à l'indigène et on lui demande de payer en espèces, quoi qu'il achète...

Dr Roberts: Qui vous empêche d'acheter à crédit ? Vous voulez dire que les marchands ici ne vous font pas crédit?

- C'est le cas, monsieur.

Tout ce que je peux dire c'est qu'ils doivent être bizarres, ces gens-là ?

— Eh bien monsieur, ils ne font pas du tout confiance aux indigènes. ${ }^{64}$

Un autre problème est que les commerçants africains ne peuvent pas se permettre d'acheter les marchandises en gros et qu'ils sont donc contraints de payer à des prix plus élevés que les commerçants blancs et indiens, et souvent pour des marchandises de qualité inférieure. Comme l'explique Jeremiah Marupe, représentant des commerçants d'Alexandra :

La valeur de ces marchandises est de loin inférieure à que ce que nous sommes contraints de payer à l'achat [...] Si vous allez dans un magasin européen ou indien et que vous achetez ces articles, vous les payez moins cher que dans mon magasin. ${ }^{65}$

Pour illustrer sa remarque, il présente plusieurs articles aux commissaires :

[Un] commerçant doit acheter des marchandises de qualité inférieure. J'ai avec moi un certain nombre d'articles que je voudrais montrer à la Commission. (Le témoin produit des articles d'épicerie, etc.). Un pain, qui est consommé par une personne - c'est-à-dire un pain fait à partir de cette farine -, n'est pas aussi bénéfique à cette personne qu'un pain fait d'une farine de meilleure qualité. ${ }^{6}$

Marupe explique que les commerçants africains d'Alexandra achètent, au prix de deux shillings la douzaine, des pains d'une livre de qualité inférieure chez les boulangers blancs de Johannesburg. Ils n'ont pas d'autre choix que de les revendre à prix coûtant (c'est-à-dire à 2 pence le pain), étant donné qu'un pain de qualité supérieure est proposé au même prix en ville. En offrant à prix coûtant des produits de base, tels que du pain, les épiciers africains des

\footnotetext{
62 South Africa, Report of the Native Economic Commission, 1930-1932, Pretoria, Government Printer UG22-1932, paragr. 951.

63 /bid, paragr. 951.

64 Témoignage de Cronje Bynight Mbolokwe, in Minutes of Oral Evidence, op. cit., p. 2157.

65 Témoignage de Jeremiah Klein Marupe, in Minutes of Oral Evidence, op. cit., pp. 8290-8291.

66 Ibid.
} 
townships espèrent attirer les membres de leur communauté dans leurs commerces plutôt que de risquer de voir leur clientèle s'approvisionner dans les magasins urbains. La stratégie mise en œuvre pour faire des bénéfices est alors de diviser les articles achetés aux grossistes pour les revendre en petites quantités. Par exemple, des sacs de thé, de café ou de sucre peuvent être répartis dans des petits paquets et se vendre à six pence chacun; quant au pain, on peut le vendre à un penny la tranche. Pour un penny, on peut aussi acquérir une bougie ou une cigarette, quelques boutons ou une longueur de ruban. Cette solution permet de générer des ventes avec de petites marges bénéficiaires, ce qui constitue une solution pratique face au problème de la pauvreté générale des habitants des townships qui ne peuvent acheter qu'en toutes petites quantités. Mais la possibilité à long terme de bâtir un commerce et d'accroître ses bénéfices demeure limitée : comme l'observe presque trente ans plus tard l'auteur d'une enquête sur les commerçants africains, " [l]a plupart des achats du ménage sont effectués par les enfants; il y a donc peu de chance d'exploiter le discernement du client ou de vendre un autre produit lorsque l'article désiré n'est pas disponible. Les ventes se comptent en pence plutôt qu'en livres ${ }^{67}{ }^{\prime}$.

Des données sur les revenus des commerçants sédentaires africains, comparés à d'autres professions, proviennent d'une étude conduite en 1940. Elle porte sur «les revenus et dépenses de 987 familles africaines vivant dans les quatre townships noirs de Johannesburg (Pimville, Western Native Township, Eastern Native Township et Orlando) ${ }^{68} »$. Les six commerçants de l'échantillon ont un revenu mensuel moyen de 9 livres et 7 shillings, alors que le salaire mensuel moyen masculin est de 4 livres et 2 shillings et que le revenu mensuel moyen d'une famille - y compris l'argent gagné par les femmes qui lavent du linge ou exercent d'autres activités - est de 5 livres, 6 shillings et 8 pence. L'étude constate que les dépenses mensuelles des familles dépassent souvent leur revenu, ce qui les entraine dans une spirale d'endettement: $40 \%$ des résidents du Eastern Native Township et $83 \%$ des habitants d'Orlando ont ainsi des arriérés de loyer.

En dépit de revenus moyens plus élevés, les commerçants africains ne sont donc souvent guère plus prospères que leurs voisins. Certes, ils paient les grossistes en espèces à prix fort, mais ils ont des frais généraux importants qu'ils déduisent de tout bénéfice potentiel. Comme l'explique à l'époque l'une d'entre eux :

Mon mari paie $4 £$ de loyer mensuel pour son magasin, et $6 £$ par an pour le droit d'exercer son activité (licence). Comme nous avons cinq enfants, tous scolarisés, il ne peut pas payer avant le mois février, et puis la licence lui a coûté $6 £$ et 10 shillings. Nous devons dépenser chaque shilling dès que nous nous en voyons un. Nous ne pouvons pas mettre de l'argent de côté. Le loyer du magasin et de la maison ne nous laisse rien. Mon mari a payé le loyer hier après que Madame $\mathrm{H}$. [l'employeuse de l'épouse] m'a payée. Je fais du lavage deux jours par semaine, parce que cela veut dire plus d'argent, mais vous savez, ma santé n'est pas bonne et je ne peux pas en faire plus. $^{69}$

\footnotetext{
67 UW, AD1974/5.1.1, Records of the South African Institute of Race Relations. Part III: «African Trading Rights. Correspondence 》, Reyburn Lawrence, "Training for African Traders », SAIRR memorandum RR/ 52/60, 2 mars 1960.

68 Janisch Miriam (1941), A Survey of African Income and Expenditure in 987 Families in Johannesburg 1940, (pamphlet) Johannesburg, Non-European and Native Affairs Department.

${ }^{69}$ Cité dans Janisch M., A Survey of African Income and Expenditure..., op. cit., p. 5.
} 
Dans l'ensemble, le constat le plus frappant qui émerge de cette étude de 1940 est la misère extrême de la plupart des résidents des townships: plus de $90 \%$ du revenu des familles est « dépensé pour le strict nécessaire ${ }^{70}$ ».

Tableau $n^{\circ} 3$ : répartition des dépenses d'un ménage de township ${ }^{71}$

\begin{tabular}{|l|c|}
\hline \multicolumn{1}{|c|}{ Article } & en \% du revenu total \\
\hline Nourriture & 49,2 \\
\hline Loyer & 18,4 \\
\hline Vêtements & 5,3 \\
\hline Carburant et lumière & 13,1 \\
\hline Transport & 6 \\
\hline Frais scolaires & 0,6 \\
\hline Frais relatifs aux activités religieuses & 0,8 \\
\hline Autres dépenses & 72 \\
\hline
\end{tabular}

L'étude permet de constater que les principaux articles alimentaires achetés dans les magasins locaux sont la farine de maïs, la viande, le pain, le riz, le sucre et le lait, ainsi que de petites quantités d'articles tels que des sardines, de la confiture, des pommes de terre, des choux, des oignons séchés, des tomates, du thé et du cafép ${ }^{73}$. Cette liste démontre qu'il y a eu des changements significatifs dans le régime alimentaire traditionnel des Africains des campagnes par rapport à celui des urbains. D'après Ellen Hellmann, la nourriture de base à la campagne consiste en du sorgho servi avec " un mets salé ou des achards » et supplémenté en lait frais, en lait fermenté (aması) et en une bouillie liquide de farine de maïs (marewu) ; la viande est un mets rare, réservé aux occasions festives. En revanche, les aliments de base du régime alimentaire urbain incluent de la farine de maïs, du pain, du ragoût de viande (avec ajout de pommes de terre et d'oignons), du thé ou du café. Comme l'explique Hellmann, «[1]a différence notable entre le régime des indigènes urbains et celui des ruraux est que le premier se caractérise par la prédominance de la viande, le manque de légumes verts, l'omission presque totale de fruits et l'absence de lait frais ${ }^{74}$ ». Alors que le thé, le café, le sucre et le pain sont considérés comme une partie essentielle du régime alimentaire en ville, les articles tels que la confiture et les sardines restent un «luxe rare». En ville, le lait frais a été remplacé par

\footnotetext{
70 Ibid, p. 25.

71 lbid.

72 Cette catégorie inclut les impôts, les assurances telles que celles des frais d'obsèques, les achats à crédit, les loisirs.

73 Janisch M., Table 2, p. 30. Une analyse de Dr F. W. Fox de I'Institut sud-africain de la Recherche médicale a constaté des carences nutritionnelles importantes dans ce régime alimentaire, avec une moyenne calorique par jour de 1827 , comparée à la moyenne " optimale » requise de 3500 calories pour un adulte.

74 Hellmann E., « Urban Native Food in Johannesburg... », pp. 280-281.
} 
du lait condensé, moins cher, plus sucré et pouvant se conserver plus longtemps, alors que le riz est utilisé « de plus en plus largement ${ }^{75} »$.

Hellmann souligne également un changement spectaculaire dans les habitudes alimentaires associées à la vie urbaine. On passe de deux repas par jour à la campagne, consommés en milieu de matinée et en fin d'après-midi, à trois repas par jour dans les zones urbaines, selon le modèle européen : petit-déjeuner, déjeuner et dîner en fin de soirée. Les heures des repas sont largement imposées par les horaires de travail dans les agglomérations : les Africains des townships mangent tôt le matin avant de partir au travail, prennent leur déjeuner en ville en milieu de journée et dînent le soir après leur retour. Il est devenu la norme pour les familles des townships urbains de manger ensemble, alors que, traditionnellement, dans les zones rurales, femmes et hommes mangent séparément ${ }^{76}$.

\section{Les commerçants africains face aux transformations des communautés noires urbaines au temps de l'apartheid}

Après le début de l'apartheid, mis en place en 1948, les autorités municipales de Johannesburg établissent en 1954 le principe d'une attribution des locaux commerciaux sur une base ethnique dans les townships, même si une enquête de 1960 consacrée aux commerçants africains permet de constater que cette politique n'a pas encore été réellement mise en œuvre ${ }^{77}$. Une autre décision, prise en 1954 et ratifiée en août 1959 par la Commission des Affaires non européennes et du Logement (Non-European Affairs and Housing Committee) est celle « d'attribuer des locaux commerciaux dans les townships sur la base de quotas déterminés par le nombre de familles qu'un commerce desservirait ${ }^{78} »$. Les quotas étaient :

Un épicier pour environ 250 familles

Un boucher pour environ 400 familles

Un marchand de fruits et légumes pour environ 1000 familles

Un poissonnier pour environ 1000 familles

Une crémerie pour environ 1000 familles $^{79}$

Comme l'indique Lawrence Reyburn, la distribution des commerces à travers les townships reste désordonnée en 1960 :

Ce n'est qu'à Meadowlands et Diepkloof, dans les townships du sud-ouest, que l'on trouve de véritables zones commerciales, où tout ce qui est nécessaire pour la maison peut être acheté dans plusieurs magasins regroupés dans une zone commodément centrale. La comparaison avec les townships municipaux est frappante car, dans ces derniers, les magasins sont éparpillés sur une

\footnotetext{
75 Ibid, p. 282.

76 Ibid, p. 280

77 Reyburn Lawrence (1960), African Traders. Their Position and Problems in Johannesburg's South Western Townships. A Fact Paper, Johannesburg, South African Institute for Race Relations, 6, Annexe III, pp. 42-43.

78 lbid.

79 Ibid.
} 
grande étendue et la personne qui fait ses courses est obligée, dans certaines zones, de parcourir beaucoup de chemin si elle veut faire tous ses achats. ${ }^{80}$

En novembre 1955, le Secrétaire des Affaires indigènes, William Eiselen, signe une directive : les commerçants africains («bantous ») ne sont plus autorisés à occuper des locaux commerciaux dans les zones urbaines en dehors des townships noirs. De nombreux commerçants africains du centre de Johannesburg, ainsi que ceux qui louent des locaux à des propriétaires principalement métis, indiens et chinois dans les townships de la Zone ouest (Sophiatown et Newclare), sont alors notifiés de quitter les lieux ${ }^{81}$. C'est un coup fatal pour eux, qui y avaient fait du commerce des décennies durant. The Bantu World cite l'exemple de Andries Phaloane, vendeur de poisson frit à Sophiatown depuis 1938. L'équipement électrique, dont deux chambres de réfrigération et une cuisinière électrique, dans lequel il avait investi et qui vaut plusieurs centaines de livres, ne sert plus à rien dans le township sans électricité où il est contraint de déménager ${ }^{82}$. Le SAIRR estime qu'environ 35 à 40 commerçants africains détenteurs de permis à Johannesburg sont ainsi déplacés, dont les trois quarts exploitaient des « restaurants indigènes »; 157 commerces africains sans permis établis en ville doivent eux aussi fermer ${ }^{83}$. Cette nouvelle politique coupe aussi ces commerces d'une large part de leur clientèle africaine, comme l'explique un éditorial du World:

Les Africains qui travaillent en ville auront toujours besoin de se nourrir à l'heure du déjeuner, d'aller chez le coiffeur, de se faire prendre en photo et de faire imprimer des billets de concert. Ce ne sera pas pratique pour eux d'avoir à quitter le centre et d'aller dans les townships pour faire tout cela. L'éloignement des commerçants africains n'est-il pas simplement une autre manière de transférer leur clientèle vers ceux qui ont l'autorisation de faire du commerce en ville ? ${ }^{84}$

En réponse, Eiselen insiste sur le fait que ces déplacements sont dans l'intérêt des commerçants africains prospères, puisqu'ils bénéficieront $d^{\prime}$ '« un régime de faveur dans l'attribution des lieux de commerce » dans les townships noirs :

Ma thèse est que cela leur ouvre un champ de possibilités illimitées, beaucoup plus précieuses que les quelques opportunités isolées qu'ils peuvent perdre en fin de compte, à la suite de la limitation de leurs activités dans des zones réservées à d'autres groupes raciaux. ${ }^{85}$

Toutefois, après le passage de la Loi sur la Promotion de l'autonomie bantoue (Promotion of Bantu Self-Government Act) en 1959, le ministre de l'Administration et du développement bantous déclare: "Les hommes d'affaires non blancs devront déménager dans des zones bantoues dès qu'ils auront accumulé assez de capital pour établir des commerces importants $^{86}$ ». Cette déclaration est suivie d'une circulaire du Secrétaire de l'Administration et

\footnotetext{
80 Ibid.

81 "Most Bantu Traders will have to get out by 1956. Only those in African premises can stay », Bantu World, 3 décembre 1955 ;

"Bombshell for Pretoria Traders: Told to get out by January", The World, 11 janvier 1956.

82 "Most Bantu Traders will have to get out by 1956... », art. cité.

83 UW, AD1947, Archives de I'Institut sud-africain des Relations raciales, III partie : "African Trading Rights. Correspondence ", Southern Transvaal Regional Office, SAIIR to Quintin Whyte, Director, SAIRR, 22 novembre 1955.

84 «Editorial », The World, 4 janvier 1956.

85 UW, AD1947, « African Trading Rights. Correspondence », W.W.M. Eiselen to Quintin Whyte, 6 janvier 1956.

86 Discours fait à Vereeniging, rapporté dans le Rand Daily Mail, 26 octobre 1959.
} 
du développement bantous en février 1963, qui précise que le gouvernement a l'intention de limiter autant que possible tout futur développement du commerce africain dans les villes:

[I] ne faut pas oublier que les droits commerciaux des Bantous dans les zones blanches ne constitue pas une opportunité intrinsèque pour eux, et qu'ils ne devront être autorisés que lorsque cela s'avère nécessaire, au sein de la zone résidentielle bantoue et au profit des Bantous. ${ }^{87}$

Bien qu'elle ne recommande pas la fermeture totale des commerces africains existant dans les zones urbaines, la circulaire appelle les «Bantous aisés, les commerçants bantous et autres Bantous qualifiés " à être "soutenus et encouragés » à établir des commerces et à $s^{\prime}$ installer dans les « homelands bantous ${ }^{88}$. Comme l'observe Quintin White du SAIRR dans une lettre adressée au Cape Times:

Le gouvernement a manqué à ses promesses faites en 1955, selon lesquelles les Africains pourraient se développer entièrement dans les townships urbains, mais promet désormais aux Africains de plus grandes possibilités dans les «homelands bantous ». Sauf pour les choses indispensables au quotidien, il garantit aux commerçants blancs le monopole du grand marché africain urbain. L'Afrique du Sud n'est donc pas « une économie de marché » pour les Africains. ${ }^{89}$

L'une des victimes de ce changement de cap politique est le magasin "Vuka Africa ». II a fermé lorsque l'Eastern Native Township devient, en octobre 1962, le dernier des vieux " townships indigènes » de Johannesburg à être éliminé à la suite des recommandations d'une commission gouvernementale. La zone est reconfigurée en zone résidentielle exclusive, alors que les résidents permanents sont déplacés à Emdeni, Senaoane et Zola (les townships du sudouest les plus récents) ou relogés dans une section du township de Pimville, que les habitants appellent toujours aujourd'hui « George Goch"0 ».

Au début des années 1960, au terme de la première décennie d'apartheid, Lawrence Reyburn dirige une étude détaillée sur les commerçants africains dans les townships du sudouest de Johannesburg (ensemble renommé « Soweto » en 1963). ${ }^{91}$ Son étude identifie 1190 commerces en activité, ainsi répartis :

\footnotetext{
87 UW, AD1947, «African Trading Rights. Correspondence », circulaire n A12/1-A8/1, datée du 14 février 1963 du Secrétaire de l'Administration et du Développement bantous. Bien que les zones telles que Soweto soient, de manière générique, connues sous le nom de " townships noirs », elles étaient appelées « zones bantoues urbaines » dans le langage officiel de l'apartheid. 
Tableau $n^{\circ} 4$ : répartition des commerces dans le township de Soweto ${ }^{92}$

\begin{tabular}{|l|l|}
\hline Type de commerce & Nombre \\
\hline Épicerie et div. & 400 \\
\hline Boucherie & 243 \\
\hline Produits frais & 176 \\
\hline Restaurant \& Poisson frit & 136 \\
\hline Bois \& Charbon & 95 \\
\hline Crémerie & 46 \\
\hline Herboriste & 38 \\
\hline Teinturier & 15 \\
\hline Marchand de nouveautés et d'habillement & 14 \\
\hline Garage & 11 \\
\hline Pompes funèbres & 7 \\
\hline Maçon & 3 \\
\hline Marchand de bois & 1 \\
\hline Hôtel & 1 \\
\hline Maison de la presse & 1 \\
\hline Systèmes anti-effraction & 1 \\
\hline Marchand de tabac & 1 \\
\hline Marchand de glaces & 1190 \\
\hline TOTAL & \\
\hline
\end{tabular}

Les plus grandes concentrations de boutiques se trouvent dans les plus vieux quartiers des townships du sud-ouest. 318 commerces sont localisés dans la partie de township autour d'Orlando et de ses environs immédiats. Pimville - nouveau nom de Klipspruit - compte la deuxième plus grande concentration de commerces, avec un total de 98 . Reyburn constate que la nature des magasins varie, " de quelques entreprises prospères avec un chiffre d'affaires de plusieurs milliers de livres par mois à celles pour lesquelles un chiffre d'affaires mensuel de 20 livres était exceptionnel ». La grande majorité sont des entreprises du «type individuel où c'est le propriétaire qui achète les produits du stock, s'occupe des opérations bancaires, etc., et supervise les vendeurs ». Seuls 11 des 47 commerçants passés en revue ont l'électricité. Cela

92 Ibid, Annexe I, p. 37. Le ministère de I'Administration et du développement bantous signalait qu'il y avait 1682 commerçants africains de tous types dans les zones africaines de Johannesburg en 1959. 
constitue un problème particulier pour les bouchers, qui ont du mal à conserver leur viande fraîche :

La nécessité d'écouler immédiatement leurs stocks, ajouté à la pauvreté de leur clientèle et à l'incapacité de celle-ci de faire la distinction entre différentes coupes de viande, amène les bouchers à vendre toutes les coupes à un tarif fixe... aucun des bouchers interviewés ne faisait de différence dans leurs tarifs entre les diverses qualités ou coupes de viande. ${ }^{93}$

L'étude de Reyburn a également mis en évidence les effets du déménagement autoritaire des commerces africains du centre-ville. II constate que seul $47,2 \%$ de la nourriture, $33 \%$ des médicaments, 58,1 \% des articles ménagers, et 31,8 \% des vêtements achetés par les résidents provient de l'intérieur des townships. Sur cette base, il estime que " probablement moins d'un tiers du salaire des ouvriers africains est dépensé dans les townships, de telle sorte que le groupe des commerçants africains n'est pas celui qui gagne le plus, comme on le prétend souvent ${ }^{94} \gg$.

Pendant pratiquement toute la première moitié $d u x x^{e}$ siècle, le développement d'une classe entrepreneuriale commerçante parmi les Africains de Johannesburg est restreint et entravé par les restrictions légales imposées par les autorités municipales blanches. Celles-ci essaient de les exclure des opportunités commerciales qui s'ouvrent dans les villes. Au fur et à mesure que s'accroît une population de citadins noirs définitivement établis en ville, les commerçants africains sont confrontés à la concurrence des hommes d'affaires blancs, indiens et chinois pour le contrôle du «commerce indigène ». Si l'adoption de la loi sur les Zones urbaines indigènes de 1923 (Native Urban Areas Act) les privilégient par certains aspects - en leur assurant le monopole du «commerce indigène » dans les townships noirs -, ces commerçants sont finalement forcés de délaisser les villes et leurs centres, alors même que les citadins africains y passent la majeure partie de leur temps. Ils perdent cette bataille avec I'application impitoyable de la politique d'apartheid dans les zones urbaines durant les années 1950.

Toutefois, durant cette période, nombre de commerces africains s'établissent dans les zones urbaines du Witwatersrand et une frange, réduite mais en expansion, de commerçants africains prospères émergent dans les townships, partie intégrante de la vie urbaine noire. Nombre d'entre eux sont des leaders au sein de leurs communautés et y soutiennent financièrement des activités sociales et culturelles. Leurs magasins sont devenus des points de repère et des lieux de rencontre dans des quartiers urbains qui sont, par ailleurs, ternes, monotones et misérables ${ }^{95}$. Leur impact le plus fort dans le secteur de la production culturelle a sans doute été d'influencer de nouveaux modèles de consommation noirs urbains,

\footnotetext{
93 Ibid., pp. 7-8.

94 Ibid., p. 12

95 Le 21 mars 1960, par exemple, Robert Sobukwe, leader du Congrès Panafricain, choisit le magasin d'Ephraim Tshabalala à Mofolo comme point de rassemblement de ses partisans, avant de marcher jusqu'au commissariat d'Orlando où il se fait arrêter volontairement dans le cadre d'une tragique journée de manifestation contre les passeports intérieurs (passes), imposés aux nonBlancs.
} 
notamment pour ce qui concerne la nourriture, les cigarettes et les biens de consommation de base.

Alan Cobley

Université des West Indies, Cave Hill Campus (Barbade)

\section{Bibliographie}

ABRAHAMS Peter (1954), Tell Freedom, Londres, Faber \& Faber.

Ballantine Christopher (1993), Marabi Nights. Early South African Jazz and Vaudeville, Johannesburg, Ravan Press.

Burke Timothy (1996), Lifebuoy Mean, Lux Women. Commodification, Consumption and Cleanliness in Modern Zimbabwe, Durham and London, Duke University Press.

Cobley Alan (1997), The Rules of the Game. Struggle in Black Recreation and Social Welfare Policy in South Africa, Westport, Greenwood.

DAVENPORT Rodney (1969), «African Townsmen? South African Natives (Urban Areas) Legislation through the Years », African Affairs, 68(271), pp. 95-109.

HellmanN Ellen (1936), « Urban Native Food in Johannesburg », Africa, 9(2), pp. 277-290.

(dir.) (1949), Handbook on Race Relations in South Africa, Johannesburg, SAIIR.

HOURANI Guita (2015), « The Struggle of the Christian Lebanese for Land Ownership in South Africa ». En ligne, URL : http://maroniteinstitute.org/MARI/JMS/july00/The_Struggle.htm (consulté le 30 octobre 2020).

JANISCH Miriam (1941), A Survey of African Income and Expenditure in 987 Families in Johannesburg 1940, (pamphlet) Johannesburg, Non-European and Native Affairs Department.

KarIS Thomas et CARTER Gwendolyn (1977), From Protest to Challenge. A Documentary History of African Politics in South Africa 1882-1964, 4, Stanford, Hoover Institution Press.

Kuper Leo (1965), An African Bourgeoisie. Race, Class and Politics in South Africa, New Haven and London, Yale University Press.

KuzWayo Ellen (1985), Call Me Woman, London, The Women's Press.

Mathabane Mark (1986), Kaffir Boy. The True Story of a Black Youth's Coming of Age in Apartheid South Africa, New York, Free Press.

MphaHLELE Ezekiel (1959), Down Second Avenue, Londres, Faber \& Faber.

OdendaAl Andre (2003), The Story of an African Game. Black Cricketers and the Unmasking of One of South Africa's Greatest Myths, 1850-2003, Cape Town, David Philip.

POSEL Deborah (2010), « Races to Consume: Revisiting South Africa's History of Race, Consumption and the Struggle for Freedom », Ethnic and Racial Studies, 33(2), pp. 157-175. 
ReYBURN Lawrence (1960), African Traders. Their Position and Problems in Johannesburg's South Western Townships. A Fact Paper, Johannesburg, South African Institute for Race Relations, 6.

Rogerson C. M. (1986), «Feeding the Common People of Johannesburg, 1930-1962 », Journal of Historical Geography, 12(1), pp. 56-73.

Skota Trevor Dan Mweli (dir.) (c. 1930), The African Yearly Register. Being an Illustrated National Biographical Dictionary (Who's Who) of Black Folks in Africa, Johannesburg, R. L. Esson \& $C^{\circ}$.

South Africa, Bureau of Census and Statistics (1960), Union Statistics for Fifty Years, Pretoria, Bureau of Census and Statistics. 